\title{
Chronic subthreshold cortical stimulation for adult drug-resistant focal epilepsy: safety, feasibility, and technique
}

\author{
Panagiotis Kerezoudis, MD, ${ }^{1}$ Sanjeet S. Grewal, MD, ${ }^{1}$ Matthew Stead, MD, PhD, ${ }^{2}$ \\ Brian Nils Lundstrom, MD, PhD, ${ }^{2}$ Jeffrey W. Britton, MD, ${ }^{2}$ Cheolsu Shin, MD, ${ }^{2}$ \\ Gregory D. Cascino, MD, ${ }^{2}$ Benjamin H. Brinkmann, PhD, ${ }^{2}$ Gregory A. Worrell, MD, PhD, ${ }^{2}$ and \\ Jamie J. Van Gompel, MD'
}

Departments of ${ }^{1}$ Neurosurgery and ${ }^{2}$ Neurology, Mayo Clinic, Rochester, Minnesota

\begin{abstract}
OBJECTIVE Epilepsy surgery is effective for lesional epilepsy, but it can be associated with significant morbidity when seizures originate from eloquent cortex that is resected. Here, the objective was to describe chronic subthreshold cortical stimulation and evaluate its early surgical safety profile in adult patients with epilepsy originating from seizure foci in cortex that is not amenable to resection.
\end{abstract}

METHODS Adult patients with focal drug-resistant epilepsy underwent intracranial electroencephalography monitoring for evaluation of resection. Those with seizure foci in eloquent cortex were not candidates for resection and were offered a short therapeutic trial of continuous subthreshold cortical stimulation via intracranial monitoring electrodes. After a successful trial, electrodes were explanted and permanent stimulation hardware was implanted.

RESULTS Ten patients ( 6 males) who underwent chronic subthreshold cortical stimulation between 2014 and 2016 were included. Based on radiographic imaging, intracranial pathologies included cortical dysplasia $(n=3)$, encephalomalacia $(n=3)$, cortical tubers $(n=1)$, Rasmussen encephalitis $(n=1)$, and linear migrational anomaly $(n=1)$. The duration of intracranial monitoring ranged from 3 to 20 days. All patients experienced an uneventful postoperative course and were discharged home with a median length of stay of 10 days. No postoperative surgical complications developed (median follow-up length 7.7 months). Seizure severity and seizure frequency improved in all patients.

CONCLUSIONS The authors' institutional experience with this small group shows that chronic subthreshold cortical stimulation can be safely and effectively performed in appropriately selected patients without postoperative complications. Future investigation will provide further insight to recently published results regarding mechanism and efficacy of this novel and promising intervention.

https://thejns.org/doi/abs/10.3171/2017.5.JNS163134

KEY WORDS intractable epilepsy; medically refractory epilepsy; cortical stimulation; cortical electrodes; surgical technique

$\Lambda$ PPROXIMATELY one-third of the 3 million people in the United States with epilepsy continue to have seizures, despite taking antiseizure medications..$^{6,11}$ Resection has been established as a safe and effective treatment for these patients; ${ }^{21}$ however, epileptic foci located within eloquent cortex represent a specific challenge, as postoperative neurological morbidity might outweigh potential benefits.
Several recent studies have shown brain stimulation to be a viable strategy for select patients with intractable epilepsy. ${ }^{8,10,19}$ Many different cortical and subcortical brain regions, including the thalamus, the cerebellum, and the hippocampus, have been selected as neurostimulation targets utilizing either duty cycle $^{14}$ or responsive stimulation paradigms. ${ }^{3}$ Despite their demonstrated efficacy in randomized controlled trials, currently approved stimulation

ABBREVIATIONS CSCS = chronic subthreshold cortical stimulation; iEEG = intracranial electroencephalography; RNS = responsive neurostimulation; SOZ = seizure onset zone.

SUBMITTED December 15, 2016. ACCEPTED May 8, 2017.

INCLUDE WHEN CITING Published online October 20, 2017; DOI: 10.3171/2017.5.JNS163134. 
approaches do not often result in seizure-free outcomes., ${ }^{3,14}$ Chronic subthreshold cortical stimulation (CSCS) might represent a more effective option. Currently available responsive neurostimulation device detection algorithms ${ }^{3}$ use a low spectral bandwidth $(0.1-100 \mathrm{~Hz})^{22}$ and do not probe small spatial scales $\left(<1 \mathrm{~mm}^{3}\right)$ and abnormal microdomain oscillations,${ }^{18}$ limiting early detection. Similarly, duty cycle stimulation paradigms (e.g., 1 minute on and 5 minutes off) leave ample time for pathological rhythms to develop and evolve into large-scale seizures. We have previously published our preliminary experience with CSCS focusing on seizure impact and outcome. ${ }^{12}$ Herein, we describe the safety, feasibility and technique of CSCS in 10 adult patients.

\section{Methods}

After institutional review board approval was granted, data were obtained from the patients' medical records and operative notes. All patients were diagnosed with seizures refractory to multiple antiseizure medications and had epileptic foci residing within eloquent cortex based on intracranial electroencephalography (iEEG) monitoring and cortical stimulation mapping. A trial of focal CSCS was performed via the monitoring electrodes in each patient prior to permanent implantation, as previously described. ${ }^{12}$ Stimulated electrodes included subdural contacts and depth electrode contacts determined from prolonged iEEG to be in the seizure onset zone.

After the iEEG electrodes were explanted, permanent stimulation was performed with hardware typically used for spinal cord stimulation or deep brain stimulation, which were repurposed for implantation and stimulation targeting the detected seizure focus with CSCS. The 16-electrode PrimeAdvanced and RestoreUltra NeuroStimulators (both Medtronic) were used as the implantable pulse generators. The hardware used (all Medtronic) included $2 \times 8$ surgical leads (model 39286, length $65 \mathrm{~cm}$ ) with sixteen $4 \times 1.5-\mathrm{mm}$ rectangular contacts; DBS lead (model 3387, length $40 \mathrm{~cm}$ ) with four $1.5-\mathrm{mm}$ electrodes at the tip separated by $1.5 \mathrm{~mm}$; DBS lead (model 3389, length $40 \mathrm{~cm}$ ) with four $1.5-\mathrm{cm}$ electrodes at the tip separated by $0.5 \mathrm{~mm}$; DBS lead (model 3391, length $40 \mathrm{~cm}$ ) with four 3-mm electrodes at the tip separated by $4 \mathrm{~mm}$; Resume II lead (model 3587, length $25 \mathrm{~cm}$ ) with a conducting electrode tip. Dually tunneled (2 to 1) $40-\mathrm{cm}$ lead extensions were also used to take advantage of all 16 channels of the battery. All patients were fully informed of the procedure's benefits, risks, and the off-label use of these implants, and all consented to the operation.

\section{Results}

Ten adult patients (6 males) underwent CSCS between 2014 and 2016; 6 patients were included in a prior brief report. Patient age ranged from 19 to 56 years with seizure history duration of 2-50 years. Intracranial pathologies included the following: cortical dysplasia $(n=3)$, encephalomalacia $(n=3)$, cortical tubers $(n=1)$, Rasmussen encephalitis $(\mathrm{n}=1)$, and linear migrational anomaly $(\mathrm{n}=$ 1). The duration of intracranial monitoring ranged from 3 to 20 days, with a 10-day median length of stay. An expanded description of CSCS is included in the initial case presentation below. No postoperative surgical complications developed (median length of follow-up 7.7 months). Specifically, no infections or focal deficits from permanent implants were seen. Seizure severity and seizure frequency improved for all patients. Two were in Engel Class I (free of disabling seizures), 5 were in Class II (rare disabling seizures), and 3 were in Class III (worthwhile improvement of seizure burden; Table 1)?

\section{Case Presentations}

\section{Case 1}

A 27-year-old, left-handed woman presented with medically refractory focal dyscognitive seizures associated with behavioral and speech arrest, occasionally associated with jerking movements of the left arm. MRI revealed a likely area of focal cortical dysplasia in the right superior temporal gyrus. Prior to intracranial monitoring, there was concern that this area was very close to cortex critical for language processing, as evaluated by functional MRI. For iEEG monitoring, the patient underwent a right frontotemporoparietal craniotomy with wide subdural electrode coverage as well as penetrating depth electrodes into

TABLE 1. Summary of cases

\begin{tabular}{rrrlrrrrr}
\hline $\begin{array}{c}\text { Case } \\
\text { No. }\end{array}$ & $\begin{array}{c}\text { Age at Op } \\
\text { (yrs), Sex }\end{array}$ & $\begin{array}{c}\text { Duration of Seizure } \\
\text { History (yrs) }\end{array}$ & \multicolumn{1}{c}{ Pathology } & $\begin{array}{c}\text { Duration of } \\
\text { Monitoring (days) }\end{array}$ & $\begin{array}{c}\text { TLOS } \\
\text { (days) }\end{array}$ & $\begin{array}{c}\text { Postop } \\
\text { Complications }\end{array}$ & $\begin{array}{c}\text { FU Length } \\
\text { (mos) }\end{array}$ & $\begin{array}{c}\text { Engel } \\
\text { Class }\end{array}$ \\
\hline 1 & $27, \mathrm{~F}$ & 21 & Cortical dysplasia & 8 & 11 & None & 9 & I \\
\hline 2 & $28, \mathrm{M}$ & 16 & Cortical dysplasia \& bilat MTS & 3 & 8 & None & 8.1 & II \\
\hline 3 & $25, \mathrm{M}$ & 15 & Presumptive linear migrational anomaly & 10 & 12 & None & 6.7 & II \\
\hline 4 & $56, \mathrm{M}$ & 50 & Multiple rt-sided encephalomalacias & 3 & 9 & None & 10 & III \\
\hline 5 & $19, \mathrm{M}$ & 5 & Encephalomalacia w/ gliosis & 20 & 19 & None & 4 & I \\
\hline 6 & $19, \mathrm{~F}$ & 2 & Rt precentral deep cortical dysplasia & 6 & 9 & None & 8.9 & II \\
\hline 7 & $26, \mathrm{M}$ & 11 & Encephalomalacia & 4 & 6 & None & 5.9 & II \\
\hline 8 & $22, \mathrm{~F}$ & 19 & Cortical tubers, SENs, SEGA & 9 & 11 & None & 5.6 & III \\
\hline 9 & $39, \mathrm{~F}$ & 21 & Rasmussen encephalitis, It parietal atrophy & 3 & 6 & None & 7.3 & II \\
\hline 10 & $23, \mathrm{M}$ & 12 & None & 7 & 11 & None & 20.5 & III \\
\hline
\end{tabular}

$\mathrm{FU}=$ follow-up; MTS = mesial temporal sclerosis; SEGA = subependymal giant cell astrocytoma; SEN = subependymal nodule; TLOS = total length of stay. 
the cortical dysplasia (Fig. 1). A subdural $8 \times 8$ contact grid with was placed over the right temporal region with 4-contact anterior and posterior temporal depth electrodes targeting the anterior hippocampus/amygdala and cortical dysplasia, respectively. Two $1 \times 4$ contact subdural strips were placed posteriorly, extending radially from the anterior and inferior posterior margin of the subdural grid (Fig. 1). Grids, strips, and depth electrodes contained contacts separated by $1 \mathrm{~cm}$.

During iEEG monitoring, the most frequent interictal discharges and typical clinical seizures emanated from the midposterior portion of the grid. During direct cortical stimulation for language mapping, language function was interrupted during stimulation testing of several electrodes localized to the seizure onset zone (SOZ), precluding resection. A trial of cortical stimulation was initiated using the 4 contacts from the posterior depth electrode as well as 6 neighboring grid electrodes. Empirically, stimulation was deemed more likely effective for seizure reduction if the rate of the most frequent epileptiform interictal discharges decreased significantly during the CSCS trial. The choice of which electrodes to stimulate was influenced by cortical geometry as well as the location of the most frequent epileptiform discharges and the SOZ. After the successful CSCS trial via the iEEG monitoring electrodes, 2 depth electrodes (3387s-40 and 3389s-40, Medtronic) were placed into the superior temporal lobe and inferior parietal lobule, respectively, and connected to a Medtronic 37702 internal generator. No complications developed postoperatively, and the patient was discharged home on postoperative Day 3. In the month prior to implantation and stimulation, the patient recorded 68
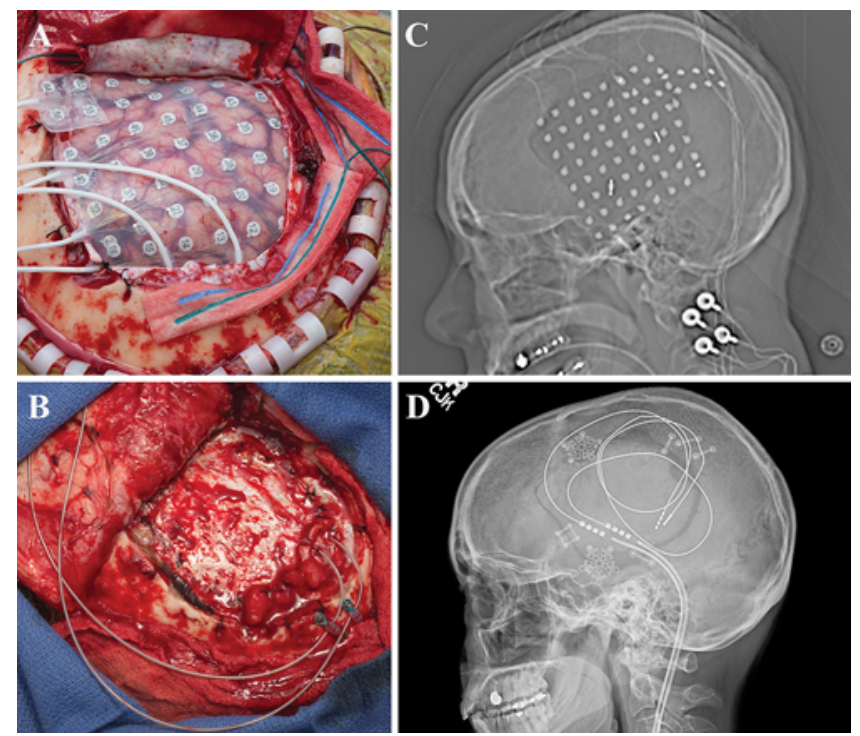

FIG. 1. Case 1. A: Intraoperative photograph obtained after the right frontotemporoparietal craniotomy with the placement of the grid and strip electrodes for electrocorticography. B: Intraoperative photograph obtained after the frontotemporoparietal craniotomy with placement of the final depth electrodes, used for chronic stimulation, into the superior temporal lobe and inferior parietal lobule. C: Lateral scout radiograph revealing the placement of the grid and multiple strip electrodes. D: Lateral scout radiograph demonstrating final placement of depth electrodes used for chronic stimulation. Figure is available in color online only. seizures, 8 of which were disabling. Following the initiation of chronic stimulation, the patient was seizure-free for 2 months and then started to have mild seizures. As of her 9-month follow-up, she continued to have intermittent mild seizures typified by unusual feelings but did not have any disabling seizures.

\section{Case 2}

A 28-year-old, right-handed man presented with a 16-year-old history of intractable focal reflex seizures involving the left lower extremity. Common triggers included standing or walking, and the patient was wheelchair bound as a result. The patient had undergone partial resection of cortical dysplasia in the parietal lobe in 2010 at an outside institution and insertion of a vagus nerve stimulator in 2014. Following these interventions, he continued to have focal reflex seizures associated with weight-bearing on his left leg, leaving him wheelchair bound. MRI findings were remarkable for encephalomalacia in the prior resection cavity and visible extension of his cortical dysplasia into the leg motor area. The patient underwent right frontal craniotomy with subdural grid insertion and interhemispheric subdural strip electrodes. iEEG demonstrated the SOZ to be concordant with eloquent motor cortex (Fig. 2). Rather than resecting the residual cortical dysplasia and leaving the patient unable to walk, a trial CSCS was performed with the implanted electrodes, and he was able to bear weight on the left leg without seizures. The patient was then returned to the operating room, and 4 leads (Resume II 3587, Medtronic) were placed, 2 interhemispherically and 2 over the right motor cortex (Fig. 2). The electrodes were connected to a Medtronic 37702 internal generator with dual tunneled (2 to 1$)$ lead extensions. The patient had an uneventful postoperative course and was discharged home on postoperative Day 3. Within the week, he was able to walk in the local shopping mall in public, which was an emotional achievement for the patient. Since commencing CSCS, he has had rare leg movements that were likely seizure related, but has otherwise been seizure free.

\section{Case 3}

A 25-year-old, right-handed man presented with a 15year history of intractable focal dyscognitive seizures manifesting as tinnitus and twitching in the right upper extremity, which often secondarily generalized. The patient's pediatric history was notable for La Crosse encephalitis. MRI findings were notable for a presumptive linear migrational anomaly deep to the left precentral gyrus. No previous surgical intervention for his epilepsy had been performed. The patient underwent a wide left frontotemporoparietal craniotomy with extensive frontal, temporal, and parietal subdural electrode coverage (Fig. 3). iEEG demonstrated 2 separate seizure foci in eloquent regions of the brain involving motor and speech. These foci were targeted, with CSCS resulting in suppression of interictal epileptiform discharges. The patient was returned to the operating room, and 2 Medtronic 39286 leads were placed over the left motor strip and attached to a Medtronic 37702 internal generator (Fig. 3). The patient was discharged home on postoperative Day 2 without any complications. 

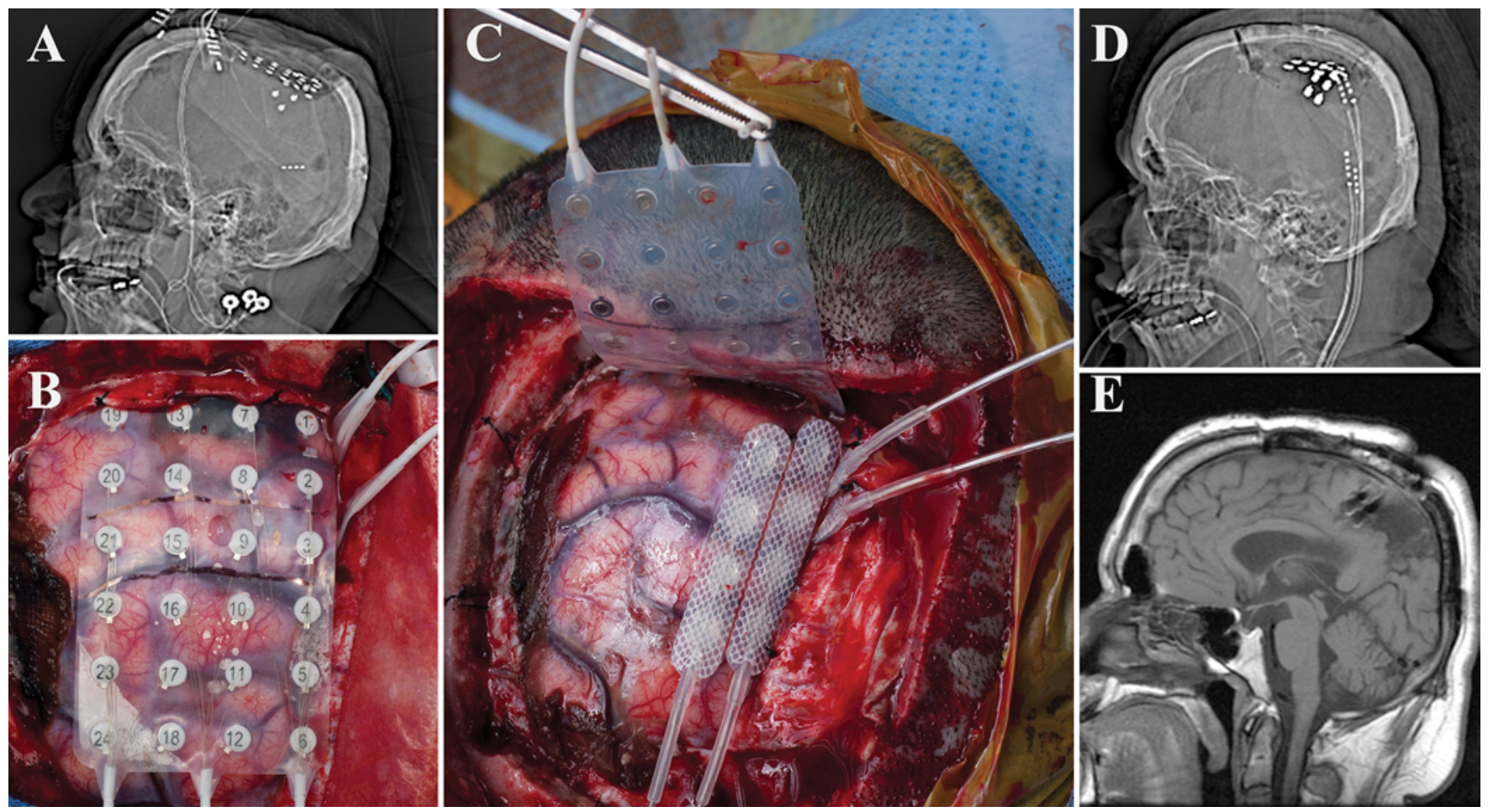

FIG. 2. Case 2. A: Lateral scout radiograph demonstrating intracranial monitoring electrodes. B: Intraoperative photograph obtained after the frontal craniotomy with a $4 \times 6$ grid and 2 strip electrodes placed interhemispherically. C: Intraoperative photograph where the $4 \times 6$ monitoring grid has been replaced with 2 Resume II electrodes sutured together over the right motor cortex, as well as 2 interhemispheric Resume II leads. These are further secured to the dura with silk sutures to ensure that they do not move. D: Lateral scout radiograph demonstrating stimulation electrodes in their final position, 2 over the motor cortex and 2 interhemispheric. E: Sagittal T1-weighted MR image demonstrating placement of interhemispheric strip electrodes over the right paracentral lobule and area of prior resection. Figure is available in color online only.

His seizure frequency decreased by approximately half, and his disabling seizures are now rare.

\section{Case 4}

A 56-year-old, right-handed man presented with a 50year history of medically intractable focal reflex seizures described as an electric shock-like sensation in the right foot and leg, followed by tonic-clonic movements that occasionally spread into the right arm. Seizures were triggered by ambulation or by placing a sock or shoe on the right foot. Notably, the patient had not worn a shoe on his right foot during his adult years because of the reflex sensory-motor seizures. The patient's pediatric history was notable for head injury due to child abuse. Neuroimaging revealed multifocal regions of encephalomalacia in the left frontal and temporal head regions. The patient underwent a left frontal craniotomy centered over the motor cortex with interhemispheric subdural strip electrodes (Fig. 4). After implantation, the patient was found to have focal seizures emanating from his leg motor area and therefore was not a candidate for resective surgery. A trial of CSCS was initiated in the hospital with the implanted electrodes. With CSCS, his usual triggers of placing a shoe on and standing on the right foot failed to activate seizure activity. The patient was subsequently returned to the operating room, and 2 Medtronic 3391 leads were placed subpially down the long axis of the leg motor area in the interhemi- spheric area; the leads were placed in this manner due to an overlying vein, which interfered with strip placement (Fig. 4). In addition, 2 strip leads (Medtronic 39286) were placed over the proximal leg region of the convexity (Fig. 4), which were connected to a Medtronic 37702 internal generator. The patient had an uncomplicated postoperative course, and he was discharged home on postoperative Day 6 . He did not have any complications, and, after years of not walking, he was able to walk out of the hospital. After about 8 months, more prominent reflex seizures returned, and at the 10-month follow-up he continues to walk.

\section{Case 5}

A 19-year-old, right-handed man presented with a 5-year history of intractable focal dyscognitive seizures, during which the patient would experience intense fear followed by clonic movements of the head to the right as well as clonic movements of the right arm and leg that sometimes progressed to generalized tonic-clonic seizures. MRI showed encephalomalacia in the left posterolateral occipital cortex. The patient underwent a left temporoparietooccipital craniotomy with extensive cortical coverage with subdural electrodes (Fig. 5). A large SOZ was identified and included critical speech and motor cortex areas. After a CSCS trial, a topectomy of the area of cortical abnormality was performed. Four Medtronic 3391 leads were then tunneled subpially into the left pos- 

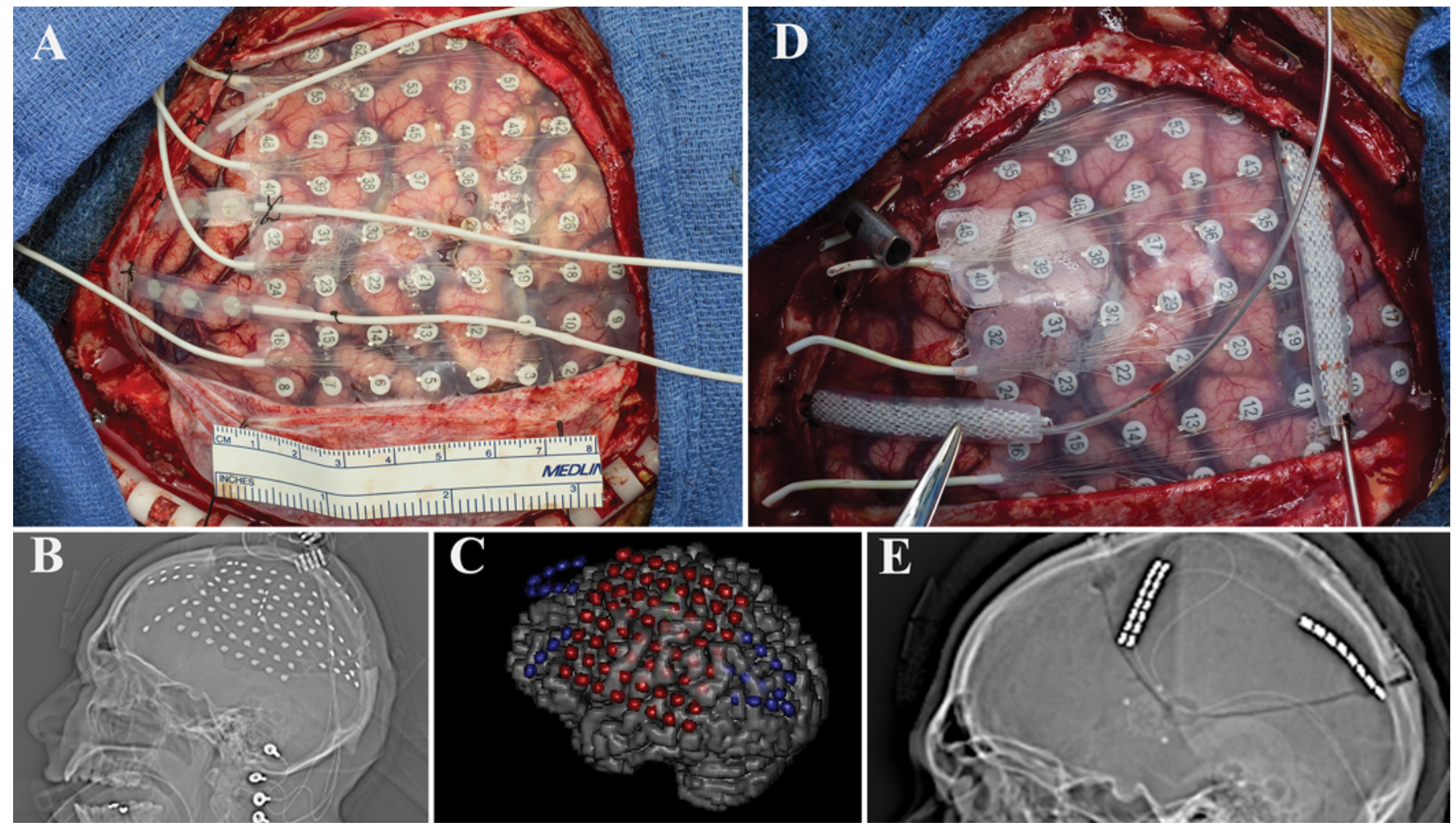

FIG. 3. Case 3. A: Intraoperative photograph obtained after the left frontotemporoparietal craniotomy, of the recording electrodes. B: Lateral scout radiograph showing the intracranial monitoring grid and strip electrodes. C: 3D reconstruction showing placement of the grid and 3 strip electrodes used for electrocorticography. D: Intraoperative photograph showing replacement of the grid and strip electrodes with 2 strip electrodes over the motor cortex and parietal seizure foci. Note that, prior to permanent placement of the electrodes, the tunneling channel is present so that this manipulation is not performed with permanent electrodes in place (metal tube in photograph) at 10 o'clock. E: Lateral scout radiograph demonstrating 2 strip electrodes over the motor cortex and parietal seizure foci. Figure is available in color online only.

terior parietal and posterior temporal region, and these were connected to a Medtronic 37702 internal generator utilizing dual lead extensions for 2-channel output (Fig. 5). The patient did not experience any postoperative complications and was discharged home on postoperative Day 4 . He continues to be seizure free 4 months after initiation of stimulation.

\section{Case 6}

A 19-year-old, right-handed woman presented with a 2-year history of intractable focal motor seizures that involved eye twitching, usually in both eyes, and which would occasionally spread to the left side of her face. The patient's medical history was unremarkable. MRI revealed a right precentral, deep cortical dysplasia. The patient underwent a right frontoparietal craniotomy with extensive cortical coverage in addition to depth electrodes placed in the facial motor region targeting the deep cortical dysplasia (Fig. 6). iEEG confirmed a seizure focus in the face motor region. A trial of CSCS was shown to suppress interictal and ictal epileptiform activity. Therefore, two $1 \times 8$ subdural electrode strips were placed over the right motor cortex and connected to a Medtronic 37702 generator (Fig. 6). The patient experienced an uneventful postoperative course and was discharged home after 3 days without complications. In the 6 weeks following initiation of CSCS, the patient experienced rare focal seizures and, over the subsequent 7 months, has been seizure free after further programming.

\section{Case 7}

A 26-year-old, right-handed man presented with an 11year history of focal dyscognitive seizures described as a feeling of cold sensation in the entire body, tunnel vision, adventitious movements in his upper and lower extremities, and loss of awareness. Neonatal history was significant for stroke in the right middle cerebral artery territory as well as apneic seizures with cyanosis soon after delivery. MRI revealed a wide area of encephalomalacia related to the perinatal stroke. He underwent stereo-EEG implantation of four 4-contact electrodes targeting the encephalomalacia and insula (Fig. 7). Although the initial implantation suggested an anterior insular focus, the patient was unwilling to consider an insular resection. CSCS was offered to the patient. Four Medtronic 3387s leads were implanted, targeting the anterior insular SOZ as determined by iEEG; 3 into the right frontal operculum terminating in the anterior insula and 1 into the anterior thalamic nucleus. These electrodes were then externalized percutaneously (Fig. 7). Subsequently, the patient underwent extraoperative scalp EEG monitoring during CSCS, which, compared with baseline, showed significant sup- 

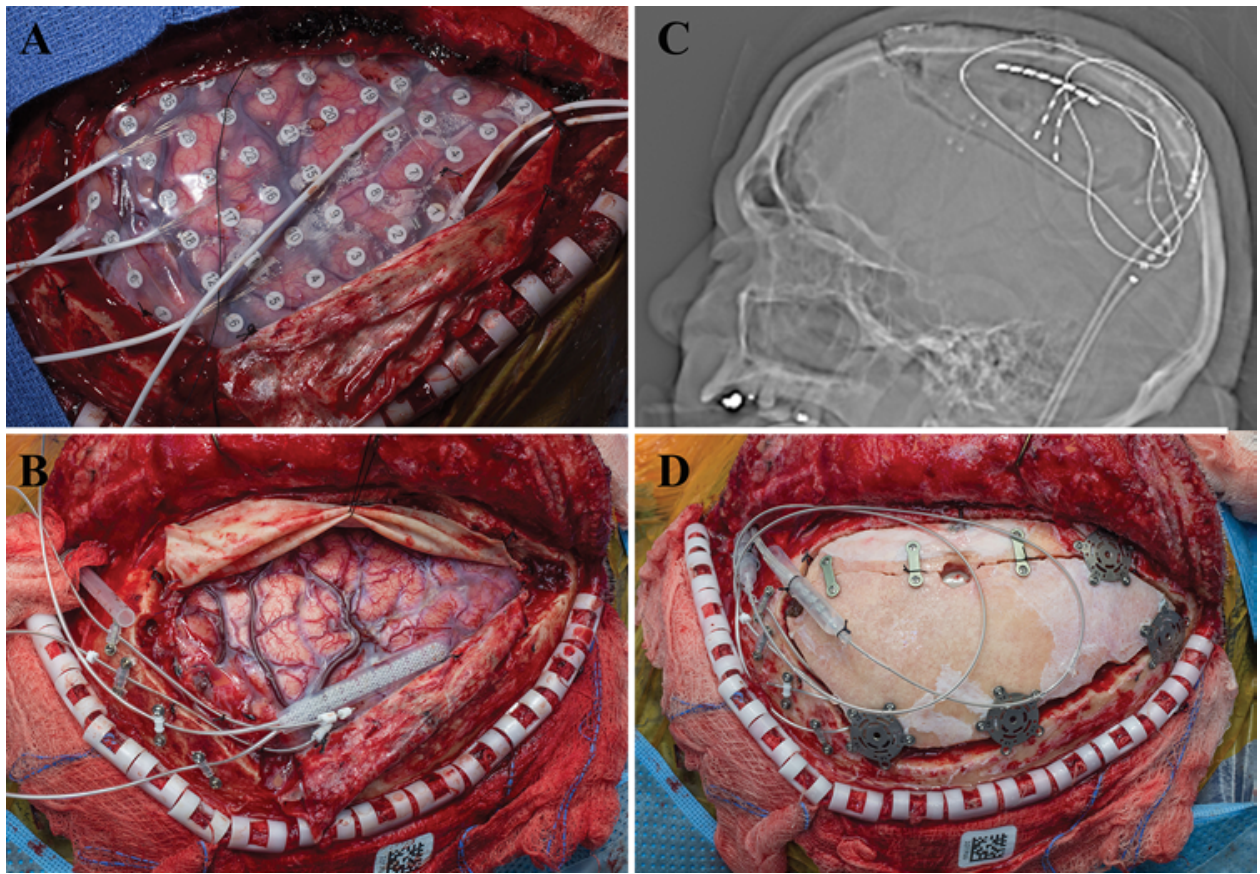

FIG. 4. Case 4. A: Intraoperative photograph obtained after the left frontal craniotomy with grid placement. B: Intraoperative photograph showing replacement of the recording electrodes with depth electrodes into the motor cortex along with a strip electrode over the region. These electrodes are stabilized at the bone edge, with a dogbone plate securing them. C and D: Lateral scout radiograph $(C)$ and intraoperative photograph $(D)$ obtained after plating of the craniotomy bone, demonstrating the lead extensions over the craniotomy site and tunneling to the left. Figure is available in color online only.

pression of interictal epileptiform discharges and seizure frequency when seizure medications were withdrawn. He was subsequently returned to the operating room where the 4 leads were internalized and connected to a Medtronic 37702 internal generator. The patient did not experience any postoperative complications and was discharged home on postoperative Day 2. In the subsequent 6 months, the patient has had 2 typical seizures, compared with clusters of up to 60 seizures about every 4 weeks prior to CSCS.

\section{Case 8}

A 22-year-old, right-handed woman with tuberous sclerosis presented with a 19-year history of daily focal dyscognitive seizures characterized by tinnitus, hand wringing automatisms, and behavioral arrest followed by aphasia and confusion. The patient also complained of nocturnal events consisting of arousals followed by confusion and semipurposeful motor activities. Her past surgical history was notable for placement of a vagus nerve stimulator in 2013 with limited seizure control. MRI showed 3 tubers, the most prominent of which enhanced after contrast administration and was located in the left posterior temporal region. The patient underwent a left frontotemporoparietal craniotomy, and 4 depth electrodes were placed in Heschl's gyrus around the cortical tuber, along with wide subdural cortical coverage (Fig. 8). Extraoperative iEEG demonstrated seizures originating from the perilesional tissue around the tuber; however, the overlying cortex was found to be critical for speech during cortical stimulation mapping. CSCS was trialed and was successful when targeting the perilesional cortex. Three Medtronic 3387 leads and one 3391 lead were placed around the tuber within Heschl's gyrus (Fig. 8). These leads were connected to a Medtronic 37702 internal generator. The patient had an uneventful postoperative course and was discharged home after 3 days. Seizures have continued, although they have reduced in frequency. The patient's family estimates an $80 \%$ improvement in seizure severity.

\section{Case 9}

A 39-year-old, right-handed woman presented with a 21-year history of intractable focal motor seizures on the right side of her face and right hand exacerbated by talking, and secondary generalized tonic-clonic seizures. The patient's pediatric history was remarkable for Rasmussen encephalitis involving the left parietal cortex. MRI was notable for left parietal atrophy and mild parietal white matter signal abnormality without any focal abnormalities. No prior surgery had been performed. The patient underwent left-sided craniotomy with placement of a $6 \times 8$ frontoparietal subdural electrode grid for monitoring (Fig. 9). Extraoperative iEEG demonstrated epileptiform activity over the left motor cortex, and clinical findings were consistent with epilepsia partialis continua. The patient was returned to the operating room 3 days after the first procedure, where 2 Medtronic 39286 leads were placed over the left primary and supplementary motor area and attached to a Medtronic 97712 internal generator (Fig. 9). The patient was discharged home on postoperative Day 6 without postoperative complications. As of 7 months postintervention, she continues to have seizures, although CSCS largely controls the facial seizures that were most bothersome to her. 

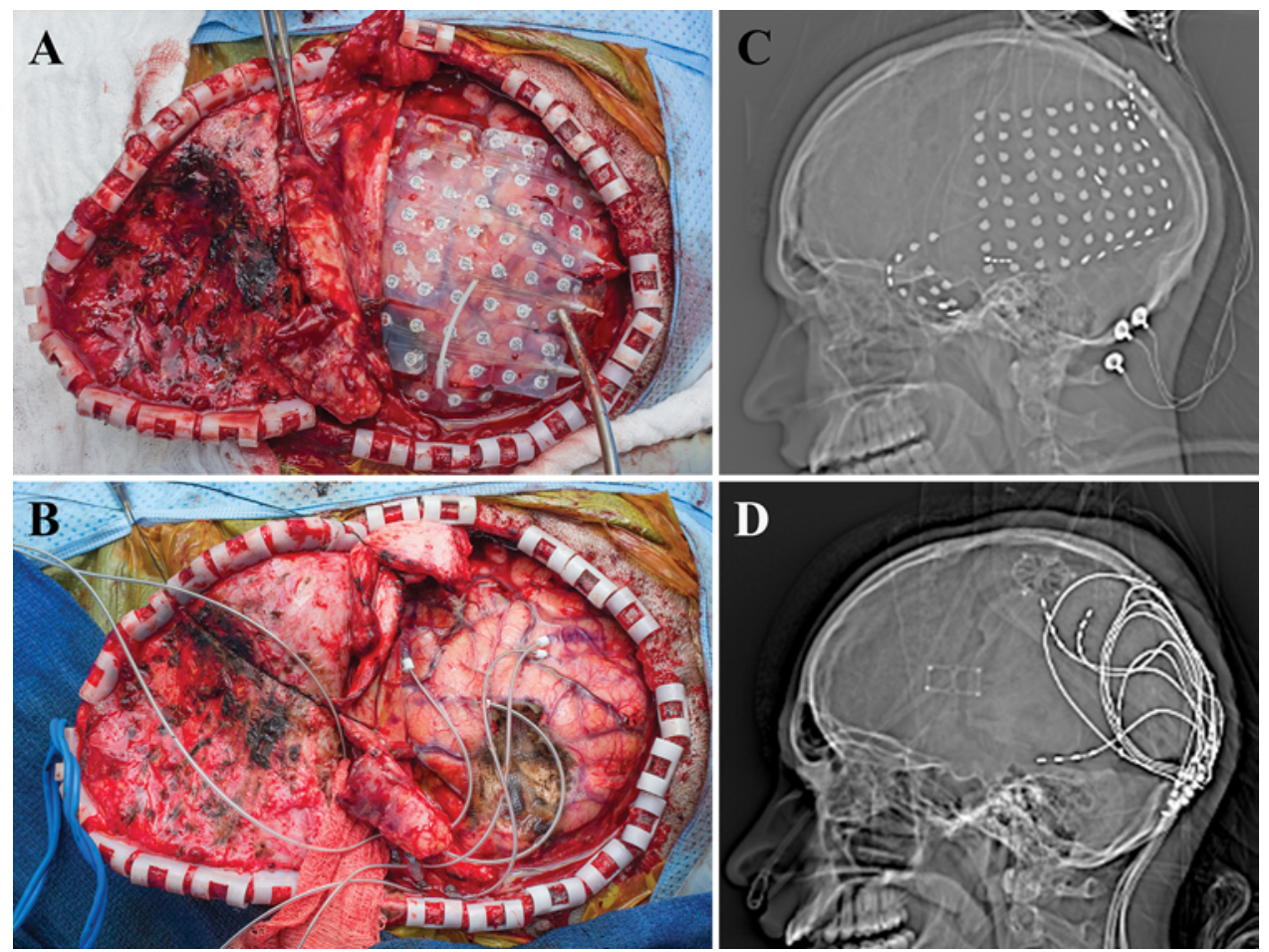

FIG. 5. Case 5. A: Intraoperative photograph obtained before the left temporoparietooccipital craniotomy with grid placement. B: Intraoperative photograph obtained after topectomy of the area of encephalomalacia along with subpial placement of the 4 model 3391 depth electrodes. Note that we placed a plastic tether to limit further insertion of the electrodes. These are further attached to the bone edge with a dogbone plate to prevent electrode migration. C: Lateral scout radiograph demonstrating placement of the monitoring grids. D: Lateral scout radiograph showing final placement of the depth electrodes. Figure is available in color online only.
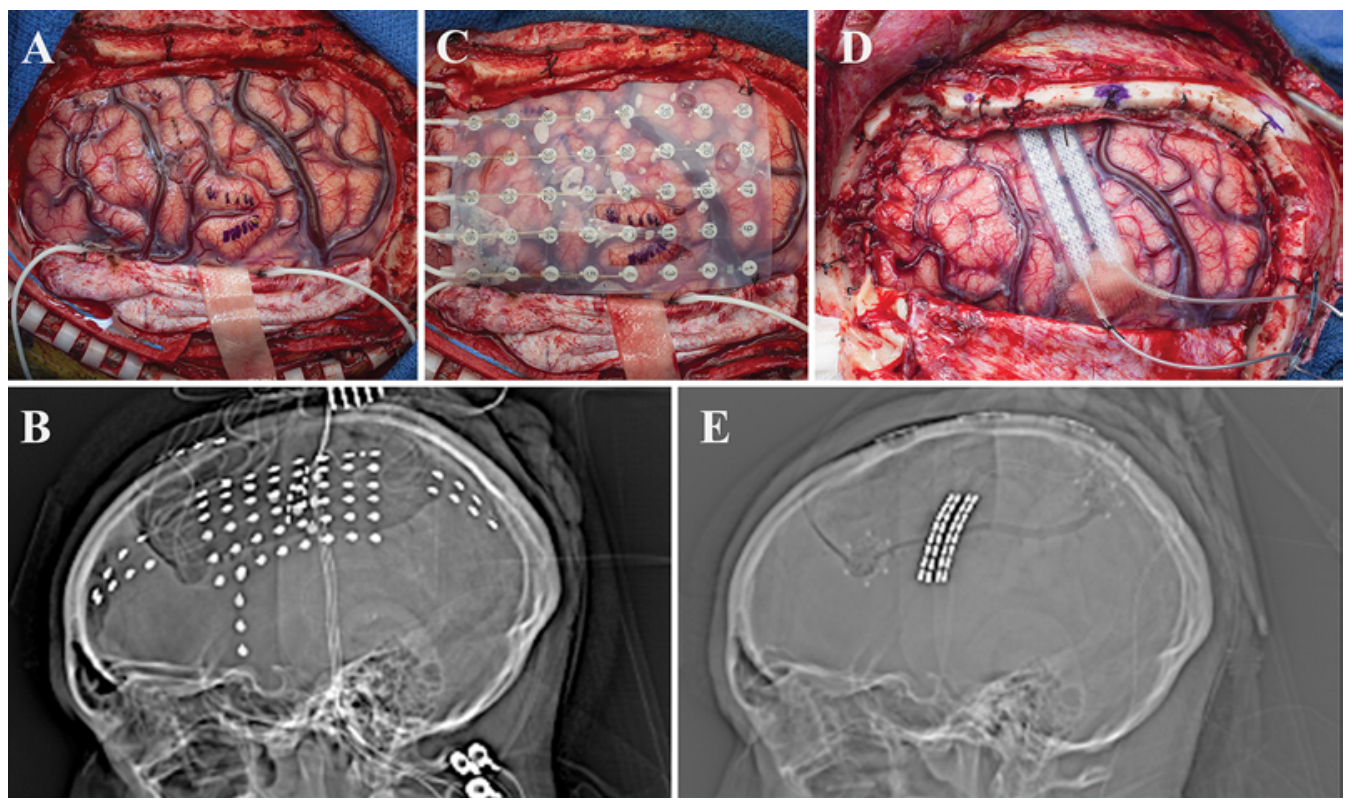

FIG. 6. Case 6. A: Intraoperative photograph obtained after the right frontoparietal craniotomy with placement of strip electrodes. B: Lateral scout radiograph showing the placement of the grid and multiple strip electrodes. C: Intraoperative photograph obtained after the right frontoparietal craniotomy with placement of strip electrodes and a large grid for coverage of the right hemisphere. D: Intraoperative photograph showing replacement of recording electrodes with 2 strip electrodes over the motor cortex used for trial stimulation. E: Lateral scout radiograph showing 2 strip electrodes over the motor cortex. Figure is available in color online only. 

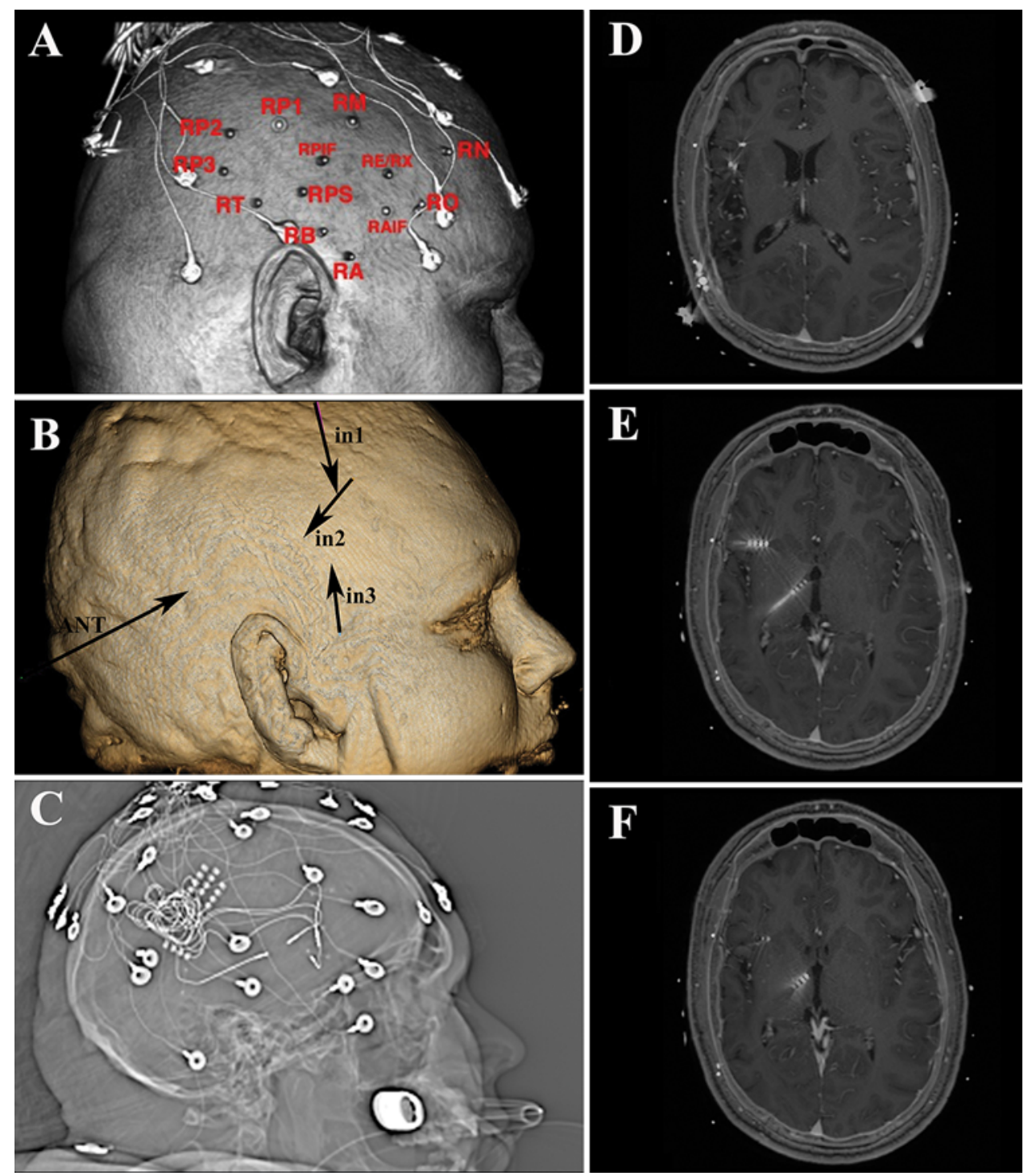

FIG. 7. Case 7. A: Scalp reconstruction showing the placement of monitoring electrodes in the region of right encephalomalacia. B: 3D skull model revealing insertion and vector for depth of the permanent depth electrodes. C: Lateral scout radiograph showing the monitoring electrodes. D-F: Axial T1-weighted MRI sequences revealing locations of the 4 permanent depth electrodes used for chronic stimulation. Figure is available in color online only.

\section{Case 10}

A 23-year-old, right-handed man presented with a 12year history of intractable focal sensory and motor seizures manifesting as electric shock-like sensation and followed by jerking of the left upper extremity. MRI did not identify any brain abnormalities or epileptogenic foci. The patient underwent right-sided frontoparietal craniotomy with extensive frontal and parietal subdural electrode monitoring (Fig. 10). Extraoperative iEEG and cortical mapping demonstrated overlap of the SOZ and right primary motor cortex. The patient was returned to the operating room after 7 days, where 4 Resume II leads were placed over the right motor strip and attached to a Medtronic 37702 internal generator (Fig. 10). The patient was discharged home on postoperative Day 11 without postoperative complications. At the 20-month follow-up, the patient reported an overall decrease of $80 \%-90 \%$ in epilepsy severity.

\section{Discussion}

Based on our institutional experience, CSCS for patients with drug-resistant epilepsy originating from eloquent cortex can be performed safely, with no apparent additional morbidity related to trial stimulation or the permanent implantation phase. At present, no complications have been reported in these adult patients. This is a critical finding, as iEEG monitoring is associated with an increased risk of infection, even without permanent hardware implantation. To minimize risk of infection in our patients, they are treated prophylactically with cefepime and vancomycin during hospitalization and with 4 weeks of cefadroxil therapy after permanent implantation. In addition, despite placement of electrodes around and into eloquent cortex, we have not experienced any temporary or permanent neurological complications. On clinical examination at the most recent follow-up, none of the patients exhibited 

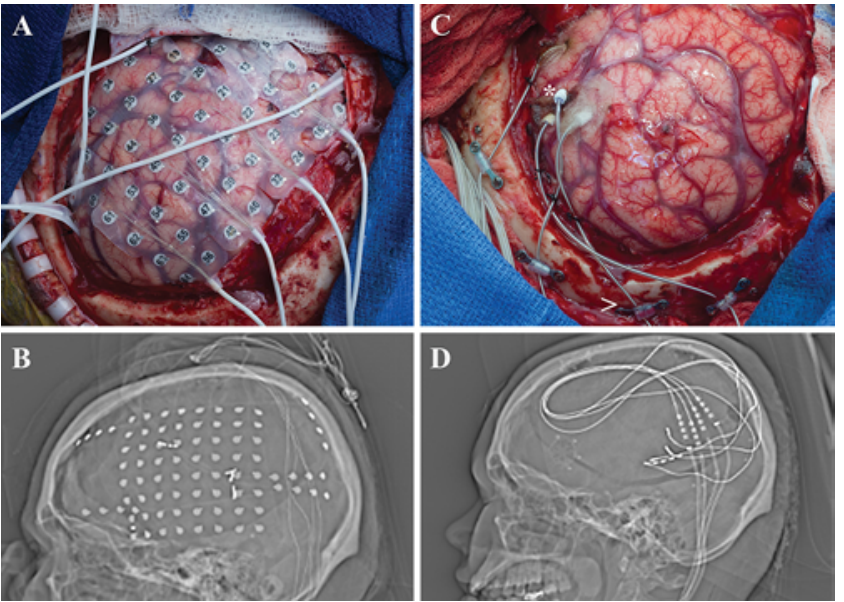

FIG. 8. Case 8. A: Intraoperative photograph obtained after the left frontotemporoparietal craniotomy with placement of the grid and multiple strip recording electrodes. B: Lateral scout radiograph showing the placement of the grid and multiple strip electrodes. C: Intraoperative photograph obtained after the left frontotemporoparietal craniotomy with placement of depth electrodes used for stimulation therapy. ${ }^{*}$ denotes the placement of the electrode into Heschl's gyrus. D: Lateral scout radiograph demonstrating the final placement of depth electrodes used for chronic stimulation. Figure is available in color online only.

any signs of focal neurological deficit (e.g., weakness or numbness) associated with the stimulated area. It is worth mentioning, however, that more-than-subtle neuropsychological sequelae could have been missed in the follow-up assessments. Furthermore, we have not encountered electrode migration over time. With an admittedly small sample size of 10 patients, we have seen an overall acceptable risk profile with this approach in which we are modifying spine and deep brain stimulation hardware for the purpose of focal cortical and subcortical CSCS. Surgical intervention for intractable epilepsy requires a careful assessment of risk and benefit. For example, resection of motor and/or sensory cortex in children with benign rolandic epilepsy is associated with Engel Class I or II outcomes in approximately $40 \%-77 \%$ with an up to $28 \%$ risk of postoperative complications. ${ }^{2}$ CSCS appears to be associated with an acceptable risk, and is generally reversible if unsuccessful; however, to date, no patient has required explantation of the hardware.

Chronic subthreshold cortical stimulation has been primarily studied in animal models. ${ }^{5}$ The first report in a patient was published by Yamamoto and colleagues in 2002 of a 31-year-old man with medically intractable mesial temporal lobe epilepsy who underwent low-frequency electrical cortical stimulation by means of subdural electrodes. ${ }^{24}$ The authors found that interictal epileptiform discharges at the seizure focus occurred less frequently after 250 seconds of stimulation. Similar results were presented by the same team in 2006, where a total of 4 patients (one with occipital cortical atrophy, one with frontal lobe tumor, one with calcified mass in the right sylvian fissure, and one with a history of perinatal asphyxia) underwent the same procedure in the absence of postoperative adverse events. ${ }^{23}$ Notably, none of the aforementioned patients received permanent implantation of stimulation
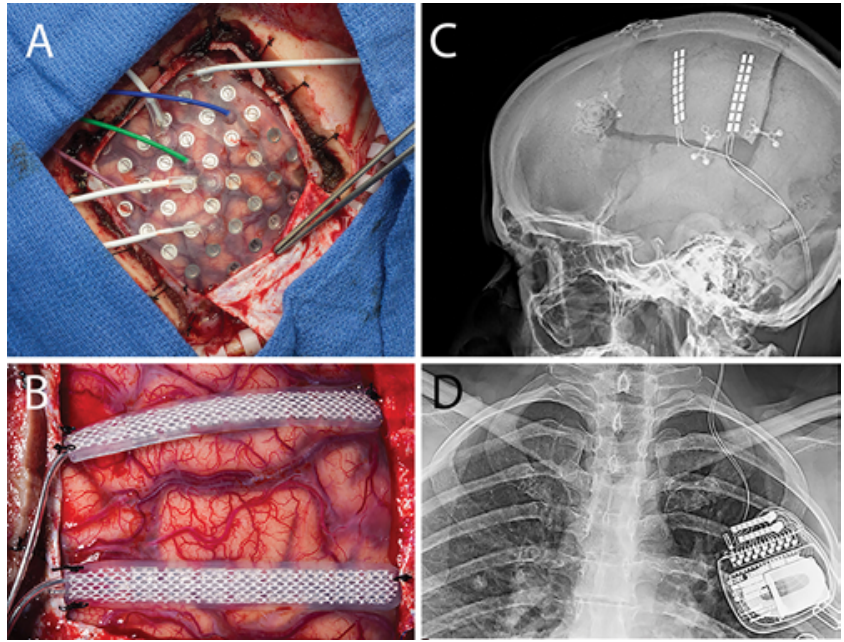

FIG. 9. Case 9. A: Intraoperative photograph showing the grid implant over the motor cortex. B: Intraoperative photograph showing 2 spinal cord stimulator strips sutured to the dura over the prior test stimulation sites. Note the hypervascularity of the cortex, consistent with the diagnosis of Rasmussen encephalitis. C: Lateral scout radiograph showing the implant. Note that in the newer spinal implants, one of the 2 electrode leads is cut as the leads exit the dura and do not require a lead extension. D: Anteroposterior chest radiograph. The spinal cord stimulator battery is implanted in the chest ipsilateral to the side of the implant. Figure is available in color online only.

hardware. Later, Velasco and colleagues reported on an adolescent and a teenager, each with nonlesional refractory motor epilepsy, who were treated with duty cycle (1 minute on $/ 4$ minutes off) therapeutic neurostimulation of the right motor area. ${ }^{20}$ In their series, neither patient experienced side effects, and seizure frequency decreased by more than $90 \%$, while motor function was preserved. Lastly, our own previously reported experience with pediatric and adult patients has demonstrated the safety, feasibility, and efficacy of CSCS.,12

Class 1 evidence for neurostimulation for epilepsy comes from the 2 landmark trials, the SANTE (Stimulation of the Anterior Nucleus of Thalamus for treatment of refractory Epilepsy) trial and the Responsive Neurostimulation (RNS) System Pivotal trial. These trials demonstrated the efficacy of neurostimulation, and that the majority of serious adverse events occur within the 1st postoperative month..$^{8,10,13}$ The SANTE trial showed a $40 \%$ reduction in seizure frequency compared with baseline in the active stimulation group versus $15 \%$ in the nonstimulated group. ${ }^{8}$ Similarly, the multicenter RNS trial showed a 38\% reduction in seizure frequency compared with the sham group. ${ }^{10,13}$ During the subsequent open-label phase of the RNS trial, there was an overall $66 \%$ reduction in seizure frequency, $56 \%$ responder rate, and improved quality of life. ${ }^{3}$ Similarly, in the open-label phase of the SANTE trial, there was a $69 \%$ reduction in seizures, a $68 \%$ responder rate, and improved quality of life..$^{14}$ However, patients infrequently experience a seizure-free outcome. In the RNS trial, $13 \%$ of patients had at least a 12-month period of seizure freedom and in the SANTE trial $16 \%$ of the patients had at least a 6-month period of seizure freedom.

According to a recent systematic review by Gooneratne 

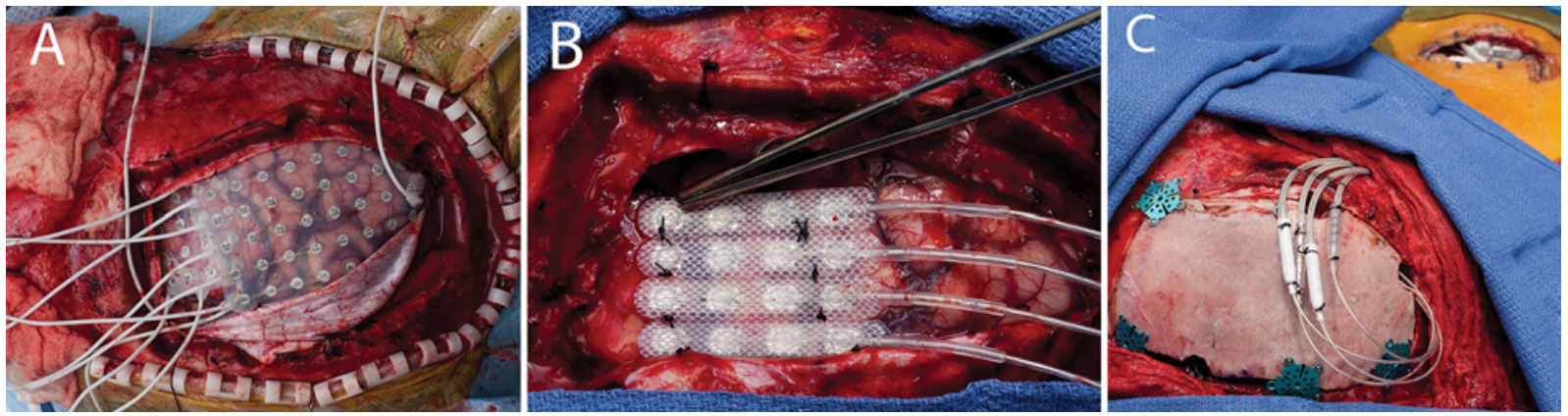

FIG. 10. Case 10. A: Grid implant. B: Final electrode configuration with Resume II leads sutured together in a row of 4 placed over the test stimulation site. C: Intraoperative photograph of the implant with the battery in place. Notice that the lead extension connectors in this circumstance are placed over the craniotomy. These connectors are typically sutured down higher on the head so that the patient does not experience discomfort when lying on them. Furthermore, the incision to the right of the field is the battery pocket. The lead extensions are typically tunneled behind the ear with the patient in pinions, which requires thought in patient positioning. Figure is available in color online only.

and colleagues, ${ }^{9}$ the most common serious adverse events associated with responsive cortical stimulation are implant site infection (9.4\%) followed by intracranial hemorrhage of any type (4.7\%). Notably, death has been observed in 11 patients so far, 7 of which have been attributed to possible, probable, or definite sudden unexpected death in epilepsy (SUDEP), ${ }^{9}$ which were not greater than expected compared with the medically intractable focal epilepsy population. ${ }^{16}$ Similarly, in the SANTE trial, device-related adverse events occurred in $35.5 \%$ of patients undergoing deep brain stimulation of the anterior thalamic nucleus. Infection and intracranial hemorrhage were observed in $10 \%$ and $4.6 \%$ of treated subjects, respectively. Although a total of 7 deaths have been reported, none of them were device related and 3 were attributed to SUDEP. ${ }^{9}$ Other less frequent device-related complications that have been described include lead damage, repeat surgery for revision of lead location, and exacerbation of seizure frequency and/ or duration. Notably, alternative options for seizures originating in eloquent brain regions, such as multiple subpial transections, appear to be less effective and pose a greater risk of neurological injury. ${ }^{17}$

CSCS presents an alternative to thalamic stimulation and RNS. In contrast to thalamic stimulation, CSCS directly targets the SOZ, based on the hypothesis that seizures can be prevented there prior to spreading. In contrast to the current FDA-approved RNS device, CSCS as implemented above avoids the complications of a skull-based device and does not necessarily preclude MRI. In contrast to RNS, CSCS is not responsive stimulation. It remains unclear to what extent the responsive aspect of current RNS systems is efficacious. Although the mechanism by which CSCS is efficacious is unknown, one possibility is that stimulation is continually decreasing cortical excitability on a relatively short time scale and is thus required chronically. It may also provide improved efficacy by virtue of continuous neuromodulation that does not depend on seizure detection, which is limited by current device sensing paradigms that use narrow spatial and spectral bandwidths, and duty-cycle stimulation paradigms that leave the epileptic network free to generate seizures during the off portion of the cycle. Optimizing the multitude of parameters involved in CSCS remains a challenge, and highlights the need for improved biomarkers of cortical excitability.

\section{Conclusions}

In the present case series, we presented our own institutional experience with 10 adult patients treated with CSCS for focal drug-resistant epilepsy involving eloquent cortex. There is morbidity risk associated with subdural grid placement (overall 2.1\%-13.6\%), including hemorrhage, extraaxial fluid and blood collections, and infection requiring additional surgical intervention in up to $3 \%$ of complications. ${ }^{1,15}$ Evidence here suggests that this procedure can be safely performed in appropriately selected patients. While the current study provides only Class 4 evidence and is limited to 10 patients with short follow-up, preliminary results demonstrate that CSCS shows promise as a safe and effective alternative to resective or ablative therapies.

\section{References}

1. Arya R, Mangano FT, Horn PS, Holland KD, Rose DF, Glauser TA: Adverse events related to extraoperative invasive EEG monitoring with subdural grid electrodes: a systematic review and meta-analysis. Epilepsia 54:828-839, 2013

2. Benifla M, Sala F Jr, Jane J, Otsubo H, Ochi A, Drake J, et al: Neurosurgical management of intractable rolandic epilepsy in children: role of resection in eloquent cortex. Clinical article. J Neurosurg Pediatr 4:199-216, 2009

3. Bergey GK, Morrell MJ, Mizrahi EM, Goldman A, King-Stephens D, Nair D, et al: Long-term treatment with responsive brain stimulation in adults with refractory partial seizures. Neurology 84:810-817, 2015

4. Child ND, Stead M, Wirrell EC, Nickels KC, Wetjen NM, Lee KH, et al: Chronic subthreshold subdural cortical stimulation for the treatment of focal epilepsy originating from eloquent cortex. Epilepsia 55:e18-e21, 2014

5. Della Paschoa OE, Kruk MR, Hamstra R, Voskuyl RA, Danhof M: Seizure patterns in kindling and cortical stimulation models of experimental epilepsy. Brain Res 770:221-227, 1997

6. Duncan JS, Sander JW, Sisodiya SM, Walker MC: Adult epilepsy. Lancet 367:1087-1100, 2006

7. Engel J Jr, Van Ness PC, Rasmussen TB, Ojemann LM: Outcome with respect to epileptic seizures, in Engel J Jr (ed): 
Surgical Treatment of the Epilepsies, ed 2. New York: Raven Press, 1993, pp 609-621

8. Fisher R, Salanova V, Witt T, Worth R, Henry T, Gross R, et al: Electrical stimulation of the anterior nucleus of thalamus for treatment of refractory epilepsy. Epilepsia 51:899-908, 2010

9. Gooneratne IK, Green AL, Dugan P, Sen A, Franzini A, Aziz $\mathrm{T}$, et al: Comparing neurostimulation technologies in refractory focal-onset epilepsy. J Neurol Neurosurg Psychiatry 87:1174-1182, 2016

10. Heck CN, King-Stephens D, Massey AD, Nair DR, Jobst $\mathrm{BC}$, Barkley GL, et al: Two-year seizure reduction in adults with medically intractable partial onset epilepsy treated with responsive neurostimulation: final results of the RNS System Pivotal trial. Epilepsia 55:432-441, 2014

11. Kwan P, Schachter SC, Brodie MJ: Drug-resistant epilepsy. N Engl J Med 365:919-926, 2011

12. Lundstrom BN, Van Gompel J, Britton J, Nickels K, Wetjen $\mathrm{N}$, Worrell G, et al: Chronic subthreshold cortical stimulation to treat focal epilepsy. JAMA Neurol 73:1370-1372, 2016

13. Morrell MJ: Responsive cortical stimulation for the treatment of medically intractable partial epilepsy. Neurology 77:1295-1304, 2011

14. Salanova V, Witt T, Worth R, Henry TR, Gross RE, Nazzaro JM, et al: Long-term efficacy and safety of thalamic stimulation for drug-resistant partial epilepsy. Neurology 84:10171025,2015

15. Schmidt RF, Wu C, Lang MJ, Soni P, Williams KA Jr, Boorman DW, et al: Complications of subdural and depth electrodes in 269 patients undergoing 317 procedures for invasive monitoring in epilepsy. Epilepsia 57:1697-1708, 2016

16. Shorvon S, Tomson T: Sudden unexpected death in epilepsy. Lancet 378:2028-2038, 2011

17. Spencer SS, Schramm J, Wyler A, O'Connor M, Orbach D, Krauss G, et al: Multiple subpial transection for intractable partial epilepsy: an international meta-analysis. Epilepsia 43:141-145, 2002

18. Stead M, Bower M, Brinkmann BH, Lee K, Marsh WR, Meyer FB, et al: Microseizures and the spatiotemporal scales of human partial epilepsy. Brain 133:2789-2797, 2010

19. Tellez-Zenteno JF, McLachlan RS, Parrent A, Kubu CS, Wiebe S: Hippocampal electrical stimulation in mesial temporal lobe epilepsy. Neurology 66:1490-1494, 2006

20. Velasco AL, Velasco F, Velasco M, María Núñez J, Trejo D,
García I: Neuromodulation of epileptic foci in patients with non-lesional refractory motor epilepsy. Int J Neural Syst 19:139-147, 2009

21. Wiebe S, Blume WT, Girvin JP, Eliasziw M: A randomized, controlled trial of surgery for temporal-lobe epilepsy. $\mathbf{N}$ Engl J Med 345:311-318, 2001

22. Worrell GA, Jerbi K, Kobayashi K, Lina JM, Zelmann R, Le Van Quyen M: Recording and analysis techniques for highfrequency oscillations. Prog Neurobiol 98:265-278, 2012

23. Yamamoto J, Ikeda A, Kinoshita M, Matsumoto R, Satow T, Takeshita K, et al: Low-frequency electric cortical stimulation decreases interictal and ictal activity in human epilepsy. Seizure 15:520-527, 2006

24. Yamamoto J, Ikeda A, Satow T, Takeshita K, Takayama M, Matsuhashi M, et al: Low-frequency electric cortical stimulation has an inhibitory effect on epileptic focus in mesial temporal lobe epilepsy. Epilepsia 43:491-495, 2002

\section{Disclosures}

Drs. Stead, Brinkmann, Worrell, and Van Gompel report receiving support from an NIH-funded public-private partnership grant (no. UH2-NS095495) between Mayo Clinic and Medtronic, and a Medtronic-supported investigational device exemption study. The patients in this report received treatment based on compassionate, off-label use with commercially available, FDA-approved devices.

\section{Author Contributions}

Conception and design: Van Gompel, Kerezoudis. Acquisition of data: Van Gompel, Kerezoudis, Grewal, Lundstrom. Analysis and interpretation of data: Van Gompel, Kerezoudis, Grewal, Lundstrom. Drafting the article: Kerezoudis, Grewal, Lundstrom. Critically revising the article: Kerezoudis, Stead, Lundstrom, Britton, Shin, Cascino, Brinkmann, Worrell. Reviewed submitted version of manuscript: Van Gompel, Stead, Britton, Shin, Cascino, Brinkmann, Worrell. Approved the final version of the manuscript on behalf of all authors: Van Gompel. Study supervision: Stead, Britton, Shin, Cascino, Worrell.

\section{Correspondence}

Jamie J. Van Gompel, Department of Neurosurgery, Mayo Clinic, 200 First St. SW, Rochester, MN 55905. email: vangompel. jamie@mayo.edu. 